TITLE:

\title{
Invariants of 3-manifolds based on conformal field theory and Heegaard splitting
}

$\operatorname{AUTHOR}(S):$

KOHNO, TOSHITAKE

\section{CITATION:}

KOHNO, TOSHITAKE. Invariants of 3-manifolds based on conformal field theory and Heegaard splitting. 数理解析研究所講究録 1991, 756: 23-32

ISSUE DATE:

1991-06

URL:

http://hdl.handle.net/2433/82151

RIGHT: 


\author{
Invariants of 3-manifolds based on conformal field theory \\ and Heegaard splitting \\ TOSHITAKE KOHNO
}

Department of Mathematics, Kyushu University, Fukuoka 812 Japan

\title{
九大理・河野俊丈
}

\section{Introduction}

The purpose of this note is to give a brief description on the construction of topological invariants of 3-manifolds by means of projectively linear representations of the mapping class group of a closed orientable surface appearing in conformal field theory. First, we give a combinatorial description of the holonomy of $S U(2)$-Wess-Zumino-Witten model. More precisely, we derive the fusing matrices, conformal dimensions and switching operators by analyzing the monodromy representation of the Knizhnik-Zamolodchikov equation, and using the fact that these data give solutions to Moore and Seiberg's polynomial equations ([12]), we construct projectively linear representations of the mapping class group on a vector space called the space of conformal blocks. Based on these representations, we define topological invariants of 3-manifolds using a Heegaard splitting. A more detailed description of this part is given in [10]. Shortly after the discovery of new 3manifold invariants due to Witten [19], Reshetikhin and Turaev [16] gave a Dehn surgery formula using representations of the quantized universal enveloping algebra $U_{q}(s l(2, \mathrm{C}))$ with $q$ a root of unity. Our approach described in this note is different from theirs.

Our principle to define 3 -manifold invariants can be applied to other class of solutions to the Moore and Seiberg's polynomial equations. It should be noted that a similar program was also proposed by Crane [2](see also [3]). In this note we focus in particular on the invariants derived from cyclic group fusion rules. It turns out that these invariants are closely related to Gocho's geometric construction based on $U(1)$ gauge theory ([4]). We also give a Dehn surgery formula for these invariants. We are planning to give a more detailed account on this subject elsewhere.

Acknowledgement; I would like to thank the members of the Euler International Mathematical Institute for their hospitality and stimulating discussions. 


\section{2. $S U(2)$-Wess-Zumino-Witten model}

Let $\Sigma_{g}$ be a closed orientable surface of genus $g$. We denote by $\mathcal{M}_{g}$ the mapping class group $\pi_{0} D i f f^{+}\left(\Sigma_{g}\right)$. We are going to associate to $\Sigma_{g}$ a finite dimensional complex vector space $Z_{K}\left(\Sigma_{g}\right)$, which is called the space of conformal blocks in $S U(2)$ Wess-ZuminoWitten model at level $K$, and then we define the action of the mapping class group on this vector space.

A marking $\mu$ of the closed orientable surface $\Sigma_{g}$ is by definition a maximal collection of disjoint, non-contractible, pairwise non-isotopic smooth circles on $\Sigma_{g}$. We associate to $\mu$ a dual trivalent graph $\gamma(\mu)$ as shown in Figure 1. We fix a positive integer $K$ called a level. Now the vector space $Z_{K}(\gamma(\mu))$ is by definition a complex vector space with basis $\left\{e_{f}\right\}$, which is in one to one correspondence with a function $f: \operatorname{edge}(\gamma(\mu)) \rightarrow$ $\{0,1 / 2,1, \cdots, K / 2\}$ satisfying

$$
\begin{aligned}
& \left|f\left(c_{1}\right)-f\left(c_{2}\right)\right| \leq f\left(c_{3}\right) \leq f\left(c_{1}\right)+f\left(c_{2}\right) \\
& f\left(c_{1}\right)+f\left(c_{2}\right)+f\left(c_{3}\right) \in \mathbf{Z} \\
& f\left(c_{1}\right)+f\left(c_{2}\right)+f\left(c_{3}\right) \leq K
\end{aligned}
$$

for the edges $c_{1}, c_{2}$ and $c_{3}$ meeting at each vertex. Let us note that the first two conditions are so-called the Clebsch-Gordan condition for $s l(2, \mathrm{C})$.
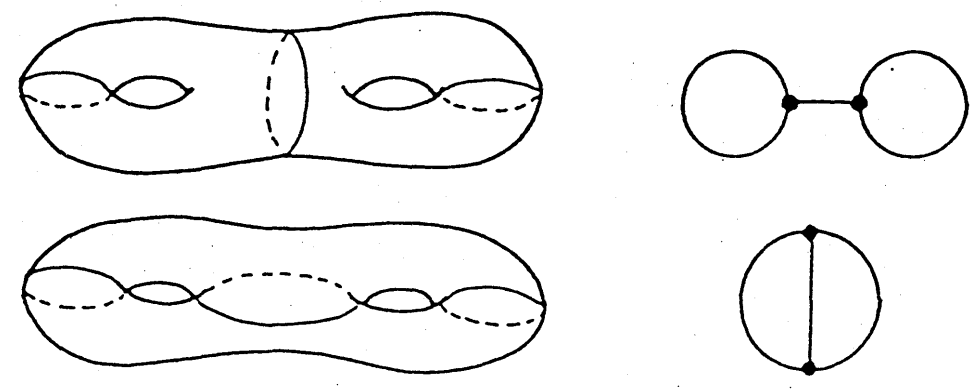

Figure 1

The basic ingredients to define the action of the mapping class group $\mathcal{M}_{g}$ on this vector space are the fusing matrices, conformal dimensions and switching operators. These data are obtained in a natural way by analyzing the monodromy representations of the following Knizhnik-Zamolodchikov differential equation ([9]). For a half integer $j$, we denote by $V_{j}$ the spin $j$ representation of $s l(2, \mathrm{C})$, which is an irreducible representation of dimension $2 j+1$. Let $j_{p}, 1 \leq p \leq K / 2$ be half integers. We put

$$
\Omega=\sum_{\mu} I_{\mu} \otimes I_{\mu}
$$

where $\left\{I_{\mu}\right\}$ is an orthonormal basis of $s l(2, \mathrm{C})$ with respect to the Cartan-Killing form. 
We define the matrices $\Omega_{i j}, 1 \leq i, j \leq n$ by

$$
\Omega_{i j}=\sum_{\mu} \pi_{i}\left(I_{\mu}\right) \pi_{j}\left(I_{\mu}\right) \in \operatorname{End}\left(V_{j_{1}} \otimes \cdots \otimes V_{j_{n}}\right)
$$

where $\pi_{i}$ and $\pi_{j}$ stand for the operation on the $i$-th and $j$-th components respectively. The Knizhnik-Zamolodchikov equation is by definition

$$
\frac{\partial \Phi}{\partial z_{i}}=\frac{1}{K+2} \sum_{j \neq i} \frac{\Omega_{i j}}{z_{i}-z_{j}} \Phi, 1 \leq i \leq \dot{n}
$$

Now we define the fusing matrix, which will be used to identify the space of conformal blocks associated with the two different "pants" decompositions as shown in Figure 2.
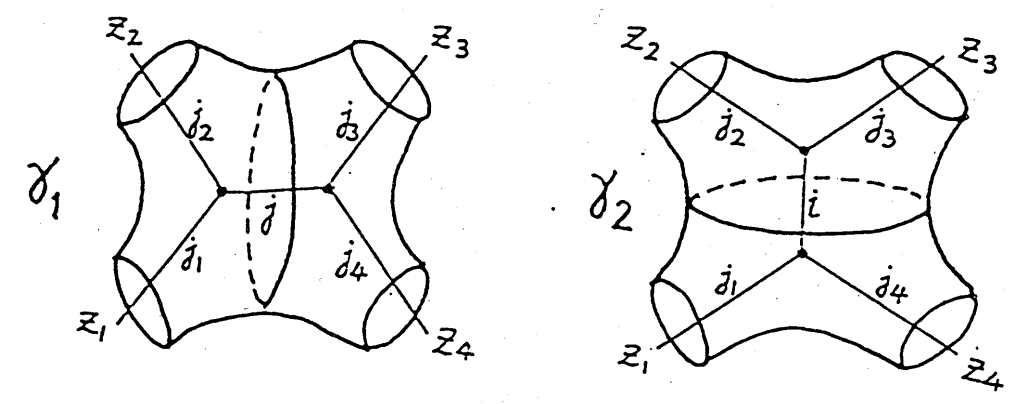

Figure 2

Let us consider the Knizhnik-Zamolodchikov equation of four variables with values in

$$
H o m_{s l(2, \mathrm{C})}\left(V_{j_{1}} \otimes V_{j_{2}} \otimes V_{j_{3}}, V_{j_{4}}\right)
$$

Let us denote by

$$
C_{j}^{j_{1} j_{2}}: V_{j_{1}} \otimes V_{j_{2}} \rightarrow V_{j}
$$

the $s l(2, \mathrm{C})$ homomorphism given by the Wigner's $3 \mathrm{j}$-symbols (see [8]). To each weighted graph depicted in Figure 2 we associate a solution of the Knizhnik-Zamolodchikov equation defined in the region $\left|z_{1}\right| \leq\left|z_{2}\right| \leq\left|z_{3}\right| \leq\left|z_{4}\right|=\infty$ in the following way. Let us suppose that the weights in the graphs in Figure 2 satisfy the admissibility condition 2-1 at each vertex. For the weighted graph $\gamma_{1}$, we consider the solution normalized around $z_{1}=z_{2}$ as

$$
\Phi_{\gamma_{1}, j}=\left(z_{2}-z_{1}\right)^{\Delta_{j}-\Delta_{j_{1}}-\Delta_{j_{2}}}\left(C_{j_{4}}^{j j_{3}} \cdot C_{j}^{j_{1} j_{2}}+\text { higher order holomorphic terms }\right) .
$$

Here $\Delta_{j}=\frac{j(j+1)}{K+2}$, which is called the conformal dimension. In a similar way, we have

$$
\Phi_{\gamma_{2}, i}=\left(z_{3}-z_{2}\right)^{\Delta_{i}-\Delta_{j_{2}}-\Delta_{j_{3}}}\left(C_{j_{4}}^{j_{1} i} \cdot C_{i}^{j_{2} j_{3}}+\text { higher order holomorphic terms }\right)
$$


normalized around $z_{2}=z_{3}$ associated with the weighted graph $\gamma_{2}$. Using an analytic continuation, it follows from a work of Tsuchiya and Kanie [17] that we have a constant matrix connecting these two solutions. We write it as

$$
\Phi_{\gamma_{1}, j}=\sum_{i} F_{i j}\left[\begin{array}{ll}
j_{2} & j_{3} \\
j_{1} & j_{4}
\end{array}\right] \Phi_{\gamma_{2}, i}
$$

The above matrix is called a fusing matrix. In a similar way, we introduce the following braiding matrix which represents the action of the half monodromy on the solution $\Phi_{\gamma_{1}, i}$ interchanging $z_{2}$ and $z_{3}$.

$B\left[\begin{array}{ll}j_{2} & j_{3} \\ j_{1} & j_{4}\end{array}\right]=F\left[\begin{array}{ll}j_{3} & j_{2} \\ j_{1} & j_{4}\end{array}\right]^{-1} \cdot \operatorname{diag}_{i}\left((-1)^{j_{2}+j_{3}-i} \exp \pi \sqrt{-1}\left(\Delta_{i}-\Delta_{j_{2}}-\Delta_{j_{3}}\right)\right) \cdot F\left[\begin{array}{ll}j_{2} & j_{3} \\ j_{1} & j_{4}\end{array}\right]$

Using a composition of fusing matrices we have an isomorphism

$$
Z_{K}\left(\gamma_{1}\right) \cong Z_{K}\left(\gamma_{2}\right)
$$

for any two dual trivalent graphs of the closed orientable surface. This isomorphism does not depend on the choice of fusing matrices involving in the above process.

Our last ingredient is the operator $S(j)$ which will be used to represent the switching operation shown in Figure 3.

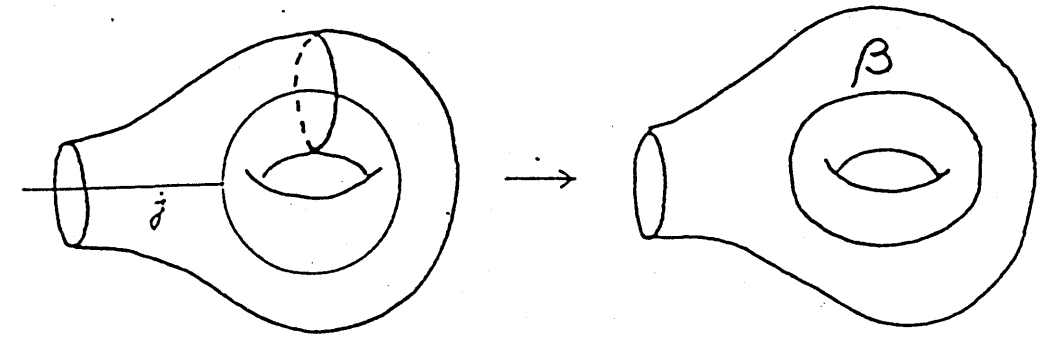

Figure 3

The operator $S(0)$ is a $k \times k$ matrix given by

$$
S(0)_{i j}=\left(\frac{2}{K+2}\right)^{\frac{1}{2}} \sin \frac{(2 i+1)(2 j+1) \pi}{K+2}, 0 \leq i, j \leq K / 2
$$

which appeared in the work of Kac-Peterson [6] to describe the modular property of the characters of the integrable highest modules of level $K$ of the affine Lie algebra of type $A_{1}^{(1)}$. The formula for $S(j)$ was obtained by $\mathrm{Li}$ and $\mathrm{Yu}[11]$ :

$$
S(j)_{p q}=\sum_{k} \exp 2 \pi \sqrt{-1}\left(\Delta_{k}-\Delta_{p}-\Delta_{q}\right) \cdot S(0)_{0 k} B_{q p}\left[\begin{array}{ll}
j & k \\
p & q
\end{array}\right], j / 2 \leq p, q \leq(K-j) / 2
$$


We put

$$
T(j)=\operatorname{diag}_{j / 2 \leq i \leq(K-j) / 2}\left(\exp 2 \pi \sqrt{-1}\left(\Delta_{i}-\frac{c}{24}\right)\right)
$$

with $c=\frac{3 K}{K+2}$. Then we have the following modular relations:

$$
\begin{aligned}
& S(j)^{2}=(-1)^{j} \exp \left(-\pi \sqrt{-1} \Delta_{j}\right) \cdot i d \\
& (S(j) T(j))^{3}=S(j)^{2}
\end{aligned}
$$

Thus we have defined the fusing matrices $F$, conformal dimensions $\Delta_{j}$ and the switching operators $S(j)$ based on the structure of the holonomy of the Knizhnik-Zamolodchikov equation. These provide solutions to the Moore and Seiberg's polynomial equations. Let us now define the action of the mapping class groups. We start with a trivalent graph $\gamma$ associated with a marking of the closed orientable surface $\Sigma_{g}$. Let $V$ be a regular neighbouhood of the graph $\gamma$ in $\mathbf{R}^{3}$ considered as a handlebody of genus $g$, and we realize $\Sigma_{g}$ as its boundary. For an edge $a$ of the graph $\gamma$, we take a disk $\Delta$ in $V$ meeting transversely with $a$ with one point and satisfying $\partial \Delta \subset \partial V$. Let $\alpha$ denote the Dehn twist about the circle $\partial \Delta$. We define the action of $\alpha$ on the vector space $Z(\gamma)$ by

$$
\alpha \cdot e_{f}=\exp \left(-2 \pi \sqrt{-1} \Delta_{f(a)}\right) e_{f}
$$

Let us recall that according to Humphries [5] the mapping class group $\mathcal{M}_{g}$ is generated by the Dehn twists $\alpha_{1}, \cdots, \alpha_{g}, \beta_{1}, \cdots, \beta_{g}, \delta$ shown in Figure 4.

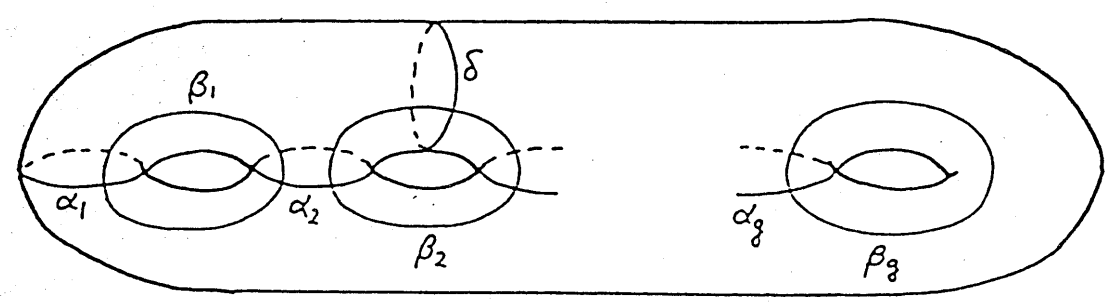

Figure 4

Considering various trivalent graphs and by identifying the associated vector spaces by fusing matrices, we can define the action of the Dehn twists $\alpha_{1}, \cdots, \alpha_{g}, \delta$. The action of the Dehn twists $\beta_{i}$ is defined in the following way. Let us go back to Figure 3 and we consider the Dehn twist $\beta$. We define the action of $\beta$ by $T(j) S(j) T(j)$. Combining with fusing matrices we can define the action of the Humphries generators. More precisely, we have 
Proposition 2.10. Let $\Omega_{K}$ denote the cyclic group generated by exp $\frac{\pi \sqrt{-1}}{4} c \cdot i d$. Then, the above construction defines a well-defined homomorphism

$$
\rho_{K}: \mathcal{M}_{g} \longrightarrow G L\left(Z_{K}(\gamma)\right) / \Omega_{K}
$$

Remark. After a suitable normalization of solutions of the Knizhnik-Zamolodchikov equation, it is known that the fusing matrices are expressed as the $q-6 j-$ symbols at $q=\exp \frac{2 \pi \sqrt{-1}}{K+2}$ (see [1], [8] and [22]). Hence we can also start from these $q-6 j-$ symbol in a purely algebraic way and we might avoid the above analytic construction related to the holonomy of the Knizhnik-Zamolodchikov equation. In [18], Turaev and Viro gave a different construction of invariants using $q-6 j$-symbols and a triangulation.

Now we are in position to define our 3-manifold invariants. Let $M$ be a closed oriented 3-manifold. It is known that $M$ admits a Heegaard splitting. Namely, there exists a handlebody $V_{1}$ and its second copy $V_{2}$ such that $M$ is obtained from $V_{1}$ and $V_{2}$ by attaching their boundaries by some $h \in \mathcal{M}_{g}$. Let us denote by $e_{0}$ a member of the basis of $Z_{K}(\gamma)$ corresponding to the weight $f$ such that $f(a)=0$ for any edge $a$. We define the $(0,0)$-entry $\rho_{K}(h)_{00}$ by

$$
\rho_{K}(h) e_{0}=\rho_{K}(h)_{00} e_{0}+\sum_{f \neq 0} \rho_{K}(h)_{f, 0} e_{f}
$$

We put

$$
\phi_{K}(M)=\left(\left(\frac{2}{K+2}\right)^{1 / 2} \sin \frac{\pi}{K+2}\right)^{-g} \rho_{K}(h)_{00}
$$

We have the following theorem.

Theorem 2.13 [10]. Let $M_{1}$ and $M_{2}$ be closed oriented 3-manifolds. If there exists an orientation preserving homeomorphism $M_{1} \cong M_{2}$, then we have

$$
\phi_{K}\left(M_{1}\right)=\phi_{K}\left(M_{2}\right)
$$

in $\mathrm{C}^{*} / \Omega_{K} \cup\{0\}$. 


\section{3. $Z / k Z$ fusion rules}

In this section, we discuss a model associated with the group algebra of a finite cyclic group. Let $k$ be a positive integer. For a closed orientable surface $\Sigma$ we construct a vector space $Z_{k}(\Sigma)$ in the following way. Let $\gamma$ be a directed graph associated with a pants decomposition of $\Sigma$ depicted as in Figure 1 . The vector space $Z_{k}(\Sigma)$ has a basis which is in one to one correspondence with weights $f: e d g e(\gamma) \rightarrow \mathrm{Z} / k \mathbf{Z}$ such that for each vertex the sum of weights corresponding to the "ingoing" edges is congruent to the sum of weights corresponding to the "outgoing" edges modulo $k$. Here we use the convention that an edge with weight $x$ is identified with the edge having the opposite direction with the weight $-x$. We see that the vector space $Z_{k}(\Sigma)$ is naturally isomorphic to the tensor product $V^{g}$ with a $k$ dimensional complex vector space, where $g$ denotes the genus of $\Sigma$.

Now we describe the action of the mapping class group $\mathcal{M}_{g}$ on $Z_{k}(\Sigma)$ using a solution of the polynomial equations due to Moore and Seiberg ([12] Appendix E) associated with $\mathrm{Z} / k \mathbf{Z}$ fusion rules. Let $m$ be a positive integer such that $m$ and $k$ are relatively prime and we suppose that $m$ is even if $k$ is odd. We put

$$
\Delta_{x}=\frac{m x^{2}}{2 k}, \quad x \in \mathrm{Z} / k \mathrm{Z}
$$

$$
S_{x y}=\frac{1}{\sqrt{k}} \exp 2 \pi \sqrt{-1}\left(-\Delta_{x+y}+\Delta_{x}+\Delta_{y}\right), \quad x, y \in \mathrm{Z} / k \mathrm{Z}
$$

Let $T$ be a diagonal matrix defined by

$$
T=\operatorname{diag}_{0 \leq x \leq k-1}\left(\exp 2 \pi \sqrt{-1} \Delta_{x}\right)
$$

One can check that the above matrices $S$ and $T$ satisfy the modular relations

$$
\begin{aligned}
S^{2} & =C \\
(S T)^{3} & =\xi(m, k) S^{2}
\end{aligned}
$$

where $C$ is the duality matrix defined by $C_{x y}=\delta_{x,-y}$ and $\xi(m, k)$ is the Gauss sum

$$
\xi(m, k)=\frac{1}{\sqrt{k}} \sum_{0 \leq x \leq k-1} \exp \frac{\pi \sqrt{-1} m x^{2}}{k}
$$

which is known to be an eighth root of unity. We introduce the fusing matrices as

$$
F_{g_{2}+g_{3}, g_{1}+g_{2}}\left[\begin{array}{cc}
g_{2} & g_{3} \\
g_{1} & g_{1}+g_{2}+g_{3}
\end{array}\right]=1
$$


for any $g_{1}, g_{2}$ and $g_{3}$ in $\mathbf{Z} / k \mathbf{Z}$.

By means of the above data, one can construct an action of the mapping class group $\mathcal{M}_{g}$ on $Z_{k}(\Sigma)$ as in the previous section. More precisely, we obtain a projectively linear representation

$$
\varphi_{m k}: \mathcal{M}_{g} \longrightarrow G L\left(V^{\otimes g}\right) /<\xi(m, k)>
$$

where the image of Dehn twists is described in the following way. We put $U=S T S, W=$ $T^{-1} \otimes T^{-1}$ and we adapt the notation for Dehn twists in the previous section. We set

$$
\begin{gathered}
\varphi_{m k}\left(\alpha_{1}\right)=T_{1}^{-1}=T^{-1} \otimes \cdots \otimes 1 \\
\varphi_{m k}\left(\alpha_{2}\right)=W_{12}, \cdots, \varphi_{m k}\left(\alpha_{g}\right)=W_{g-1, g} \\
\varphi_{m k}\left(\delta_{2}\right)=T_{2}^{-1}=1 \otimes T^{-1} \otimes \cdots \otimes 1 \\
\varphi_{m k}\left(\beta_{1}\right)=U_{1}, \cdots, \varphi_{m k}\left(\beta_{g}\right)=U_{g}
\end{gathered}
$$

Here the symbol $W_{k, k+1}, 1 \leq k \leq g-1$, stands for the operation of $W$ on the $k$-th and $(k+1)$-st components of the tensor product $V^{\otimes g}$. One can show that the above representation factors through $S p(2 g, \mathrm{Z})$.

Let $M$ be a closed oriented 3-manifold obtained as a Heegaard decomposition $V_{1} \cup_{h} V_{2}$, where $V_{1}$ and $V_{2}$ are handlebodies of genus $g$. As in the previous section we consider the $(0,0)$-component $\varphi_{m k}(h)_{00}$. We have the following theorem

Theorem 3.5. We put

$$
I_{m k}(M)=\sqrt{k^{-g}} \varphi_{m k}(h)_{00}
$$

Then, $I_{m k}(M)$ is a topological invariant of $M$.

In the case $m=1$ and $k$ is even, the above invariant was discovered by Gocho [4] from a geometric viewpoint. In fact he constructed a vector bundle over the Siegel upper half plane with a projectively flat connection whose holonomy gives the above representation of $S p(2 g, \mathrm{Z})$.

Now we describe the Dehn surgery formula of the invariant $I_{m k}$. Let us suppose that the closed oriented 3-manifold $M$ is obtained from the Dehn surgery on a framed link $L$ with $n$ components in $S^{3}$. Let $A$ be the linking matrix whose diagonal entries are given by the framing. We denote by $\sigma$ the signature of the linking matrix $A$. Using the above notations, we have the following theorem.

Theorem 3.6. We put

$$
J_{m k}(M)=\sqrt{k^{-n}} \xi(m, k)^{-\sigma} \sum_{h \in(Z / k \cdot Z)^{n}} \exp \left(\frac{\pi \sqrt{-1} m}{k} t h A h\right)
$$


Then, $J_{m k}$ is a topological invariant of $M$. Moreover, the invariant $I_{m k}$ computes this invariant up to some power of the Gauss sum $\xi(m, k)$.

Remark. The invariant $J_{m k}$ can be written as the state sum

$$
\xi(m, k)^{-\sigma} \sum_{\lambda} S_{0, \lambda(1)} \cdots S_{0, \lambda(n)} F(L, \lambda)
$$

for any $\lambda:\{1, \cdots, n\} \rightarrow \mathbf{Z} / k \mathbf{Z}$, where $F(L, f)$ denotes the product for all crossing points in the link diagram given by

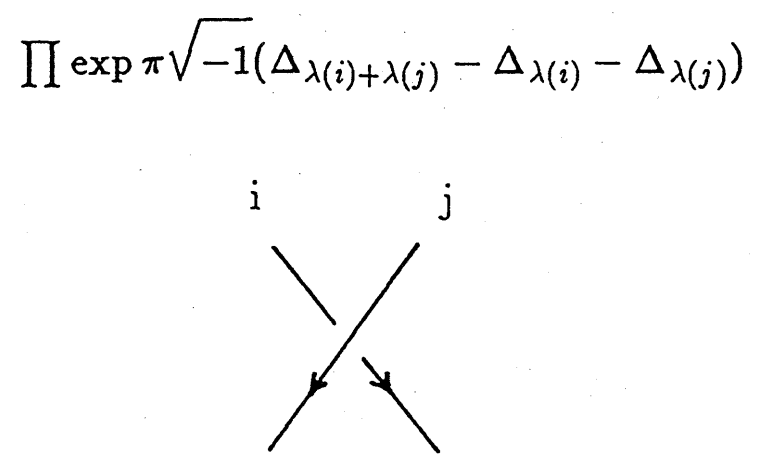

Figure 5

Here to each crossing point of $i$-th and $j$-th components we associate the weight as shown in Figure 5 and we take the product for all crossing points. The Dehn surgery formula corresponding to the case of Gocho's invariant was discussed by Ohtsuki [15]. We observe that the case $k=2$ coincides with the Reshetikhin-Turaev invariant for $r=3$. Generalizing the investigation due to Kirby and Melvin [7], Ohtsuki showed that the absolute value of $J_{1 k}$ is equal to the square root of the number of elements in $H^{1}(M ; \mathbf{Z} / k \mathbf{Z})$ if we do not have $\alpha \in H^{1}(M ; \mathbf{Z} / k \mathbf{Z})$ such that $\alpha \cup \alpha \cup \alpha \neq 0$ and is equal to 0 otherwise. In the case $k$ is even, we have a slightly different representation of $S p(2 g, \mathrm{Z})$ by putting

$$
\Delta_{x}=\frac{m x^{2}}{2 k}+\frac{x}{2}
$$

and by replacing the above Gauss sum by

$$
\delta(m, k)=\frac{1}{\sqrt{k}} \sum_{0 \leq x \leq k-1}(-1)^{x} \exp \frac{\pi \sqrt{-1} m}{k} x^{2}
$$

We have a similar construction and the resulting Dehn surgery formula

$$
\sqrt{k^{-n}} \delta(m, k)^{-\sigma} \sum_{h \in(Z / k Z)^{n}}(-1)^{<\operatorname{diag} A, h>} \exp \left(\frac{\pi \sqrt{-1} m}{k} t h A h\right)
$$


was introduced by Murakami and Okada [14] related to the cyclotomic invariants for links discovered in [13] based on the IRF model due to Kashiwara and Miwa. Here $<\operatorname{diag} A, h>$ stands for $\sum_{i} A_{i i} h_{i}$.

\section{REFERENCES}

[1] L. Alvarez-Gaumé, G. Sierra and C. Gomez, Topics in conformal field theory, Physics and Mathematics of Strings, World Scientific (1990).

[2] L. Crane, Topology of 3-manifolds and conformal field theories, preprint, Yale University (1989).

[3] S. E. Capell, R. Lee and E. Y. Miller, Invariants of 3-manifolds from conformal field theory, preprint (1990).

[4] T. Gocho, The topological invariant of three-manifolds based on the $U(1)$ gauge theory, preprint, University of Tokyo (1990).

[5] S. Humphries, Generators for the mapping class group, LNM, Springer (1979), 44-47.

[6] V. G. Kac and D. H. Peterson, Infinite dimensional Lie algebras, theta functions and modular forms, Advances in Math. 53 (1984), 125-264.

[7] R. Kirby and P. Melvin, Evaluations of the 3-manifold invariants of Witten and Reshetikhin-Turaev, London Math. Soc. Lect. Notes Series 151 (1990), 101-114.

[8] A. N. Kirillov and N. Y. Reshetikhin, Representation of the algebra $U_{q}(s l(2, \mathrm{C})$, q-orthogonal polynomials and invariants of links, Infinite dimensional Lie algebras and groups, World Scientific (1988), $285-342$.

[9] V. G. Knizhnik and A. B. Zamolodchikov, Current algebra and Wess-Zumino models in two dimensions, Nucl. Phys. B247 (1984), 83-103.

[10] T, Kohno, Topological invariants for 3-manifolds using representations of mapping class groups, to appear in Topology.

[11] M. Li and M. Yu, Braiding matrices, modular transformations and topological field theories in $2+1$ dimensions, Comm. Math. Phys. 127 (1990), 195-224.

[12] G. Moore and N. Seiberg, Classical and quantum conformal field theory, Comm. Math. Phys. 123 (1989), 177-254.

[13] T. Kobayashi, H. Murakami and J. Murakami, Cyclotomic invariants for links, Proc. Japan Acad. 64 (1988), 235-238.

[14] H. Murakami and M. Okada, talk at Waseda University, Dec. 1990.

[15] T. Ohtsuki, private comminucation.

[16] N. Y. Reshetikhin and V. G. Turaev, Invariants of 3-manifolds via link polynomials and quantum groups, to appear in Invent. Math.

[17] A. Tsuchiya and Y. Kanie, Vertex operators in conformal field theory on $\mathrm{P}^{1}$ and monodromy representations of braid groups, Advanced Studies in Pure Math. 16 (1988), 297-372.

[18] V. G. Turaev and O. Y. Viro, State sum invariants of 3-manifolds and quantum 6j-symbols, preprint, LOMI (1990).

[19] E. Witten, Quantum field theory and the Jones polynomial, Comm. Math. Phys. 121 (1989), 351-399. 